\title{
Internet of Animals: On-and Off-body Propagation Analysis for Energy Efficient WBAN Design for Dairy Cows
}

\author{
Said Benaissa ${ }^{1,2}$, David Plets ${ }^{1}$, Emmeric Tanghe ${ }^{1}$, Günter Vermeeren ${ }^{1}$, Luc Martens ${ }^{1}$, Bart \\ Sonck $^{2,3}$, Frank Tuyttens ${ }^{2}$, Leen Vandaele ${ }^{2}$, Wout Joseph ${ }^{1}$ \\ ${ }^{1}$ Department of Information Technology, Ghent University/iMinds, Technologiepark-Zwijnaarde 15, 9052 Ghent, Belgium (Tel: \\ +3293314918; e-mail: wout.joseph@intec.UGent.be) \\ ${ }^{2}$ Institute for Agricultural and Fisheries Research (ILVO), Scheldeweg 68, 9090 Melle, Belgium (Tel: +3292722605; email: \\ frank.tuyttens@ilvo.vlaanderen.be) \\ ${ }^{3}$ Department of Biosystems Engineering, Faculty of Bioscience Engineering, Ghent University, Coupure links 653, 9000 Ghent, \\ Belgium (Tel: +3292722601; email: bart.sonck@ilvo.vlaanderen.be)
}

\begin{abstract}
This paper presents propagation modelling of different on-body and off-body wireless communication scenarios for dairy cows in barns at $2.4 \mathrm{GHz}$. Based on the obtained propagation models, a WBAN that monitors multiple health parameters is designed for optimal performances in terms of energy efficiency and packet error rate.
\end{abstract}

Index Terms-Dairy cow, on- and off-body path loss, cow phantom, temporal fading, wireless body area network (WBAN), energy efficieincy.

\section{INTRODUCTION}

The size of dairy cattle farms and the number of animals per stockperson are increasing. This makes the herd monitoring - especially the detection of animals that require attention (e.g., care, treatment or assistance) - a challenging task. Wireless tracking sensors could be effectively used on herds of dairy cows to monitor their health and welfare [1], [2].

When cows are equipped with sensors, multiple health parameters (e.g., temperature from the udder or ear) and activity information (e.g., movement from legs, position) could be tracked in real time. The data is then transmitted to a data collector placed on the collar. Next, this information is forwarded to a backend access point placed in the proximity of the cows and then transferred to a central data processing server. Finally, the farm manager can easily detect illnesses such as lameness and mastitis in an early stage, which would result in less suffering for the cows as well as ensuring milk yields[2].

The success of such a health care monitoring system relies on a good characterization of the on-body (e.g., udder to neck) and the off-body (e.g., neck to backend access point) wireless communications. In this paper, we address the propagation analysis of these links at $2.4 \mathrm{GHz}$. Based on this analysis, we design an energy efficient health care monitoring system for dairy cows.

The remainder of the paper is structured as follows. In Section II, the on-body propagation is presented. Section III describes the characterization of the off-body communication. Then in Section IV, the obtained results are presented and discussed. These results are used for the WBAN design performed in Section V. Finally, conclusions are drawn in Section VI.

\section{ON-BODY PROPAGATION}

\section{A. Measurement Setup}

On-body measurements were conducted using a dairy cow in a state-of-the-art research barn at the Institute for Agricultural and Fisheries Research (ILVO) in Melle, Belgium. Two ZigBee nodes were used during this experiment. A first node was programmed as a transmitter with a transmit power of $3 \mathrm{dBm}$. The second node was programmed as a receiver to capture the received signal strength indicator (RSSI) corresponding to the transmitted packets. The RSSI values are then logged with a laptop via USB interface.

\section{B. Simulation Setup}

Simulations were performed using a 3-D electromagnetic solver (SEMCAD-X) based on the finite-difference time domain (FDTD) computation method. A homogenous cow model with the same dimensions as the experimental cow was developed for the simulations with the dielectric properties of cow muscle at $2.4 \mathrm{GHz}$, relative permittivity $\varepsilon r=52.791$ and conductivity $\sigma=1.705 \mathrm{~S} / \mathrm{m}$ [3]. A maximum grid step of $2 \mathrm{~mm}$ was taken for the cow body model, which allows correct simulation of the frequency of $2.45 \mathrm{GHz}$ [4]. To model the transmitter and the receiver, the same antennas as the ZigBee motes were modelled. For the transmitter, a simple quarter-wavelength monopole with a length of $30 \mathrm{~mm}$ was simulated. The receiver was an inverted $\mathrm{F}$ antenna (IFA) with the same dimensions and design as in [5]. 


\section{On-body Scenarios}

To design a WBAN that monitors multiple health parameters, different on-body wireless communication links have to be considered. Fig. 1 shows four scenarios where information from (i) hind leg (scenario I), (ii) udder (scenario II), (iii) leg front (scenario III), and (iv) the ear (scenario IV) is forwarded to the collector node RX placed on the cow's neck.

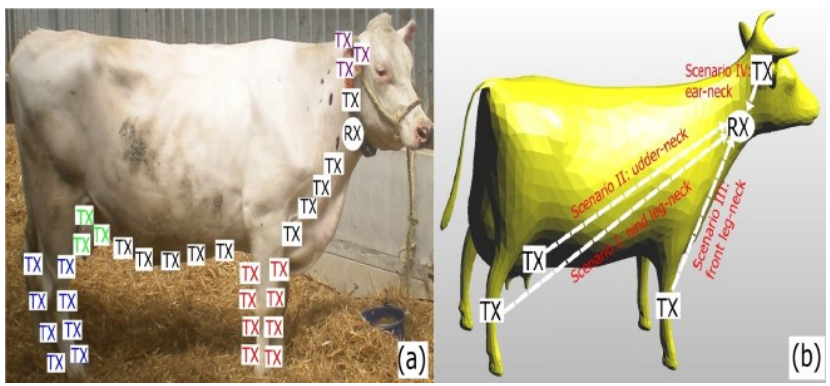

Fig.1 (a) On-body TX and RX positions. Each color represents the positions considered for each body part (i.e., blue: hind leg, green: udder, red: front leg, and purple: ear). The black positions are used for the whole body path loss calculation. (b) On-body measurements and simulations scenarios.

\section{Path loss modelling}

After obtaining an average received power $\mathrm{P}_{\mathrm{RX}}$ for each separation between TX and RX, the path loss PL is calculated as follows:

$$
\mathrm{PL}=\mathrm{P}_{\mathrm{TX}}+\mathrm{G}_{\mathrm{TX}}-\mathrm{L}_{\mathrm{TX}}-\mathrm{L}_{\mathrm{RX}}+\mathrm{G}_{\mathrm{RX}}-\mathrm{P}_{\mathrm{RX}}
$$

where $P_{T X}$ is the transmitter power $(\mathrm{dBm}), \mathrm{G}_{\mathrm{TX}}$ the transmitter antenna gain in free space $(\mathrm{dBi}), \mathrm{L}_{\mathrm{TX}}$ transmitter cable losses $(\mathrm{dB}), \mathrm{G}_{\mathrm{RX}}$ receiver antenna gain in free space $(\mathrm{dBi})$, and $\mathrm{L}_{\mathrm{RX}}$ the receiver cable losses $(\mathrm{dB})$.

The definition of the path loss given by (1) cannot be applied immediately due to the inevitable interaction between the antennas and the cow's body. Because the antennas are positioned close to the cow's body, their characteristics (i.e., radiation pattern, gain) are influenced by the body charges. In this situation, the free space antenna gain cannot be used for calculating the path loss. In literature ([6], [7]), the antenna gains are included in the WBAN path loss calculation given by (1). Thus the path loss, including the antenna gains as a part of the channel model (i.e., antenna embedded path loss), is calculated as follows:

$$
\mathrm{PL}=\mathrm{P}_{\mathrm{TX}}-\mathrm{L}_{\mathrm{TX}}-\mathrm{L}_{\mathrm{RX}}-\mathrm{P}_{\mathrm{RX}}
$$

Similarly to [6], a log-distance path loss model is proposed. The path loss can be modeled as a linear function of the logarithmic distance between the transmitter and receiver, as explained in [6]:

$$
\operatorname{PL}(d)=\operatorname{PL}\left(\mathrm{d}_{0}\right)+10 \cdot n \cdot \log \left(\mathrm{d} / \mathrm{d}_{0}\right)+\mathrm{X}_{\sigma}
$$

with $\operatorname{PL}\left(\mathrm{d}_{0}\right)$ is the path loss at reference distance $\mathrm{d}_{0}=$ $10 \mathrm{~cm}, \mathrm{n}$ the path loss exponent, $\mathrm{d}$ the separation distance between TX and RX, and $\mathrm{X}_{\sigma}$ a zero-mean Gaussian distributed variable (in $\mathrm{dB}$ ) with standard deviation $\sigma$ also in dB.

\section{OFF-BODY PROPAGATION}

To characterize the off-body propagation, two parameters have been addressed. First, the path loss, which describes the loss of power a wirelessly transmitted signal undergoes when travelling from transmit to receive side. Second, the temporal fading, which determines the variability of received power originated from movement of cows or persons in the environment.

\section{A. Path loss Measurements and Scenarios}

For the path loss measurements, two scenarios were investigated. A barn without cows and a barn with 15 cows. (i.e., one cow wearing the mote and 14 other cows moving freely in the barn).

In the first scenario (i.e., barn without cows), path loss measurements were executed with two omnidirectional vertically polarized antennas (MA431Z00). The transmitting antenna (TX) was connected to a signal generator to inject a continuous wave signal at $2.4 \mathrm{GHz}$ with a constant power of $18 \mathrm{dBm}$ (see Fig. 2). The receiving antenna (RX) was connected to a spectrum analyzer, which samples the received power level at the transmitting frequency. Sampled power values are stored on a laptop.

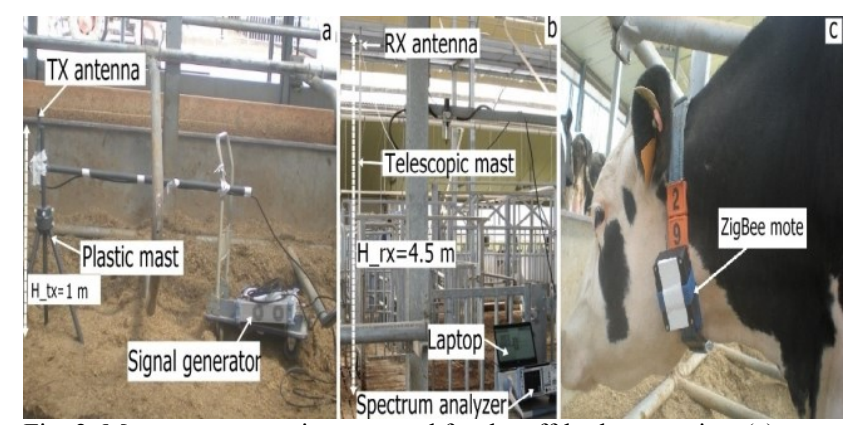

Fig. 2. Measurement equipment used for the off-body scenarios. (a)

Transmitter side for barn without cows, (b) Receiver side for both scenarios, and (c) transmitter side for barn with cows.

The receiver was placed at a fixed position with an antenna height of $4.5 \mathrm{~m}$, which is a typical height of the access points. Then, the position of the transmitter was set inside each cubicle at a height of $1 \mathrm{~m}$ above the ground. This TX height is comparable to the height of a cow's neck. The Measurements were performed for a range of distances (TXRX separation) between 7 and $27 \mathrm{~m}$ (Fig. 3).

In the second scenario, the transmitter side was replaced with one cow wearing a ZigBee mote on the collar and fifteen cows were moving freely inside the measurement area. The same TX positions as scenario 1 were investigated. For each TX-RX separation, 200 samples of the received power were logged with the spectrum analyzer and the average value was considered.

Equation (1) was used to calculate the path loss values for each TX-RX separation. Then, equation (3) was used to model the path loss as a linear function of the logarithmic distance. The reference distance was set to $1 \mathrm{~m}$ for the offbody communication. 


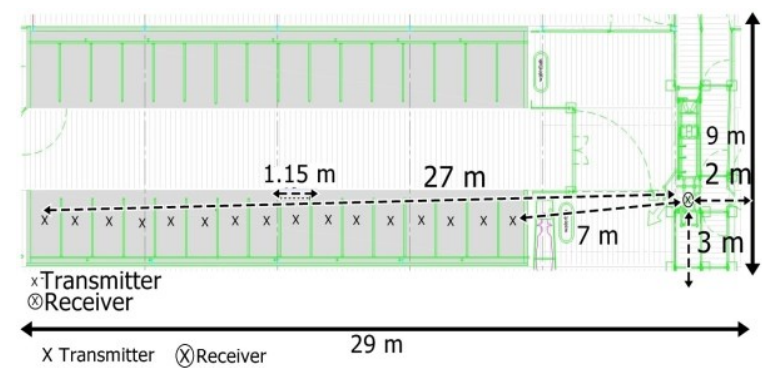

Fig. 3. TX and RX positions for the off-body path loss measurements.

\section{B. Temporal fading Measurements}

The temporal fading measurements were conducted with the same measurement setup as in the first scenario. However, the transmitter and receiver were set in stationary positions while the cows were moving freely in the measurement environment. These scenarios were set to allow the recording of received signal power variations due to the movements of the cows. At each fixed location, received power levels were recorded in a time span of 20 minutes.

To overcome the influence of temporal fading, a fade margin should be considered in the link budget analysis. The fade margin is determined by analyzing the statistics of the received power over time. In NLOS conditions, the probability density function (PDF) of the mean received signal amplitude follows a Rayleigh distribution. However, when an undisturbed multipath component (e.g., LOS component) is present, fading statistics follow a Rician distribution [8]. In our case, a dominant multipath component between transmitter and receiver was often present. Therefore, the Rician distribution is adopted to characterize the temporal fading. This assumption is validated by comparing the theoretical Rice distributions to the distribution of the measured temporal fading samples.

The Rician distribution is often described in terms of a parameter K (Rician factor) [8]. To estimate the K-factor, the method of moments proposed in [8] was used.

\section{RESUlTS AND DisCUSION}

\section{A. On-body Results}

Table I lists the average path loss values for the considered on-body scenarios. For each body part, the positions mentioned in Fig. 1 are considered in the calculation of the average path loss.

TABLE I. COMPARISON BETWEEN MEASURED AND SIMULATED AVERAGE PATH LOSS VALUES FOR THE INVESTIGATED SCENARIOS. $\delta$ IS THE ABSOLUTE DEVIATION BETWEEN MEASUREMENTS AND SIMULATIONS.

\begin{tabular}{|l|c|c|c|}
\hline \multicolumn{1}{|c|}{ Scenarios } & PL $^{\text {meas }}[\mathbf{d B}]$ & $\mathbf{P L}^{\text {sim }}[\mathbf{d B}]$ & $\boldsymbol{\delta}$ \\
\hline I- Hind leg-Neck & 64.0 & 69.4 & 5.6 \\
\hline II- Udder-Neck & 69.2 & 72.0 & 2.8 \\
\hline III- Front leg-Neck & 62.4 & 65.8 & 3.1 \\
\hline VI- Ear-Neck & 53.1 & 51.3 & 1.8 \\
\hline Average & 3.3 \\
\hline
\end{tabular}

The measured path loss $\mathrm{PL}^{\text {meas }}$ varies between $53.1 \mathrm{~dB}$ for the scenario IV (i.e., ear-neck) and $69.2 \mathrm{~dB}$ for the scenario II (i.e., udder-neck). Scenario IV has the lowest value because of the short distance between the ear and the neck of the cow (about $50 \mathrm{~cm}$ ). However, for scenario II (udder-neck), the cow's body obscures the communication between the udder and the neck, resulting in the highest path loss value. Scenarios I and III have approximately the same path loss values $(63 \mathrm{~dB})$. This result could be explained the similar influence that the legs (front and back) influence on the antennas. For the simulated path loss $\left(\mathrm{PL}^{\mathrm{sim}}\right)$, it varies between $51.3 \mathrm{~dB}$ (scenario IV) and $72.0 \mathrm{~dB}$ (scenario II). We observed an average difference of $3.3 \mathrm{~dB}$ between the measurements and the simulations. This could be explained by the homogenous cow model used for simulations.

In order to develop a path loss model for the whole body, all positions shown in Fig. 1 were considered. Then, a least squares fit was performed (fit for measurements and fit for simulations) using the path loss values for the different transmitter-receiver distances to model the path loss as a linear function of the logarithmic distance. The obtained path loss models are shown in Fig. 4 and their parameter values are listed in Table II.

The measured and simulated path loss models show a deviation of about $3 \mathrm{~dB}$, which is in accordance with the values listed in Table I.

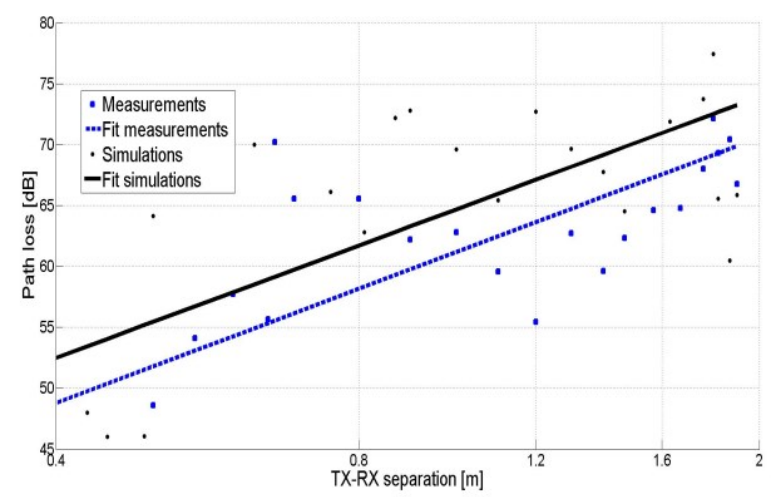

Fig. 4. Measured on-body path loss and fitted models versus distance (TX-RX separation for the whole cow's body.

TABLE II. PARAMETER VALUES OF THE ON-BODY PATH LOSS MODELS

\begin{tabular}{|l|l|l|l|l|l|}
\hline & $\mathbf{d}_{\mathbf{0}}[\mathbf{c m}]$ & $\mathbf{P L}\left(\mathbf{d}_{\mathbf{0}}\right)[\mathbf{d B}]$ & $\mathbf{n}[-]$ & $\boldsymbol{\sigma}[\mathbf{d B}]$ & $\mathbf{R}^{\mathbf{2}}[-]$ \\
\hline Measurement & 10 & 31 & 3.12 & 4.8 & 0.76 \\
\hline Simulation & 10 & 34 & 3.06 & 6.4 & 0.71 \\
\hline
\end{tabular}

\section{B. Off-body Results}

The measured path loss values and the fitted models versus log-distance (TX-RX separation) are shown in Fig. 5. As expected, the path loss of the barn without cows was lower than the path loss when the barn contains cows $(5 \mathrm{~dB})$. This is due to the cow's body shadowing (the cow wearing the mote and the other cows). 


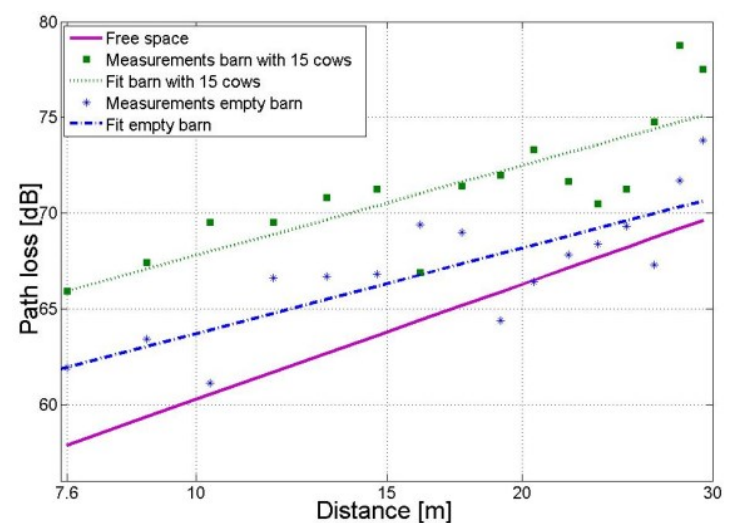

Fig. 5. Measured off-body path loss and fitted models versus distance.

Table III lists the parameter values of the obtained path loss models. The path loss exponents were lower than free space $(n=2)$ due to the presence of multipath influence inside the barn. Similar path exponents were found by [9] in indoor industrial environments at $2.4 \mathrm{GHz}$. The standard deviations were around $6 \mathrm{~dB}$. This indicates a slightly higher degree of shadow fading due to the presence of cows inside the barn. Finally, coefficients of determination greater than 0.8 were obtained, meaning that the log-normal path loss model perfectly fits the measured data.

TABLE III. PARAMETER VALUES OF THE OFF-BODY PATH LOSS MODELS

\begin{tabular}{|c|l|c|c|c|c|}
\hline Scenarios & $\mathbf{d}_{\mathbf{0}}[\mathbf{m}]$ & $\mathbf{P L}\left(\mathbf{d}_{\mathbf{0}}\right)[\mathbf{d B}]$ & $\mathbf{n}[-]$ & $\mathbf{\sigma}[\mathbf{d B}]$ & $\mathbf{R}^{2}[-]$ \\
\hline $\begin{array}{c}\text { Barn without } \\
\text { cows }\end{array}$ & 1 & 48.2 & 1.5 & 5.7 & 0.82 \\
\hline $\begin{array}{c}\text { Barn with 15 } \\
\text { cows }\end{array}$ & 1 & 53.5 & 1.8 & 6.1 & 0.85 \\
\hline
\end{tabular}

For the temporal fading, the Rician K-factor is estimated based on the moment method as presented in Section III.B. This method estimates the K-factor directly from the measured samples without need for a curve fitting operation. Based on the received power recorded over time and using the moment method, a K-factor of $10 \mathrm{~dB}$ was obtained. This value indicates a dominant path LOS component in our measurements due to the TX height $(4.5 \mathrm{~m})$. Based on the Kfactor, a fade margin associated with the temporal fading for a given outage probability is determined. The details of the calculation are explained in [10]. For an outage probability of $1 \%$ ( $99 \%$ of the time, the variation around the median will not exceed the fade margin), a fade margin of $6 \mathrm{~dB}$ should be considered in the link budget and network planning analysis in barns environment.

\section{APPLICATION}

Nodes in WBANs for dairy cows would use very small batteries with low processing and storage capabilities. Furthermore, such batteries would need to operate properly and autonomously for long periods of time without being recharged or replaced. Several choices that can impact the energy consumption, e.g., data rate (applications), complexity of routing algorithms, and programming languages. To reduce the energy consumption an efficient network topology can be a crucial factor for extending battery lifetime.

In the following, we investigate the packet error rate $(P E R)$ and the energy efficiency as a function of the transmit power for three WBAN topologies (i.e., single-hop, two-hop, and cooperative communication). The obtained path loss models (Section IV) and the energy consumption of available commercial radios (i.e., ZL70101 and nRF24L01) are considered. In Fig. 6, we propose a scenario where data collected for the udder (e.g., temperature) could be transmitted directly to the access point (backend system) in a single-hop scheme or transmitted to a relay node placed on the collar (data collector) and then forwarded to the access point. In the second case, two-hop or cooperative schemes could be used. The derivation of the energy efficiency and the packet error rate as a function of the sensor's transmit power for the single-hop, two-hop, and cooperative schemes are presented [1].

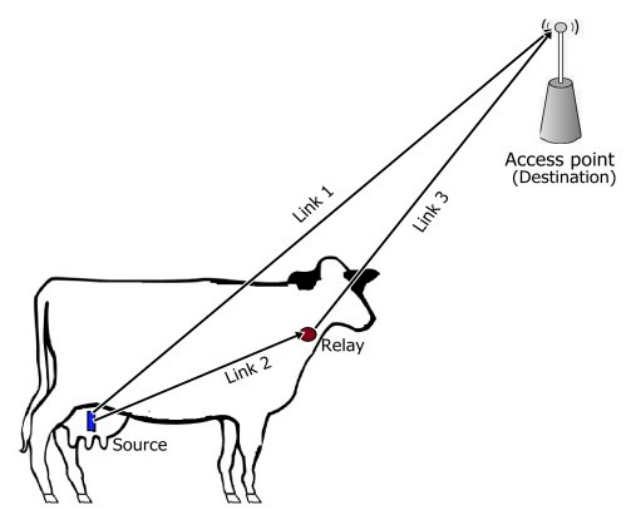

Fig. 6. Network design: the data collected from the udder (source) is transmitted directly to the access point via link 1 (sing-hop) or transmitted first to a rely node placed on the collar and then forwarded to the access point using two-hop or cooperative schemes.

The packet error rate is shown (Fig. 7) as a function of the transmit power $\left(\mathrm{P}_{\mathrm{TX}}\right)$ for a packet size of 128 bytes. We observe that the cooperative scheme presents the lower PER (highest performance) whereas the single-hop presents the highest PER. In addition, for a transmit power lower than -5 $\mathrm{dBm}$ the two-hop gives the same PER as the cooperative. For instance, to ensure a PER of $10^{-4}$, a transmit power of -4.5 $\mathrm{dBm}$ for the cooperative and two-hop schemes, and $-1 \mathrm{dBm}$ for single-hop is required. Thus, the relaying communication, either by cooperation or multi-hop, uses low power to give the same performance (PER) as the single-hop. This allows the battery lifetime of the cow sensor nodes to be extended for long-term health and welfare monitoring. It is important to note that the relaying process requires additional nodes, thus increasing the network cost.

In Fig. 8, energy efficiency is shown as a function of the sensors' transmit power. As shown in Fig. 8, a threshold transmit power exists that separates a region where a single- 


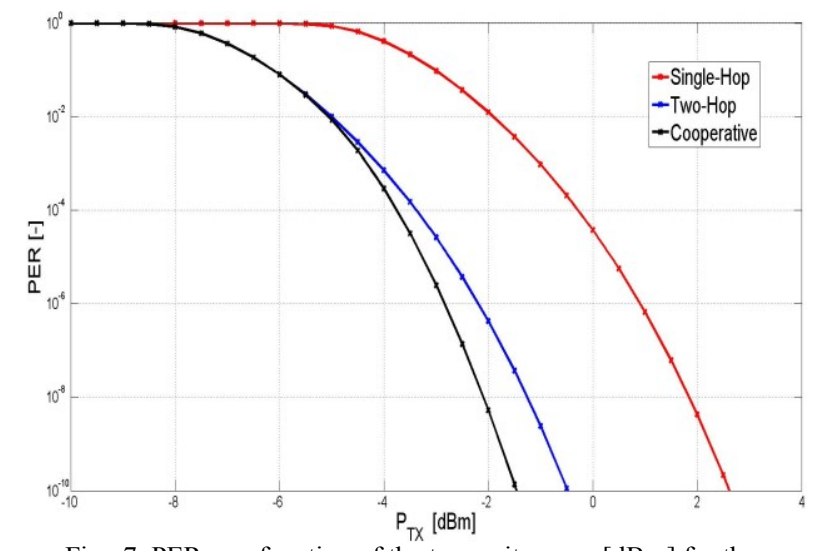

Fig. 7. PER as a function of the transmit power $[\mathrm{dBm}]$ for the investigated communication schemes (packet size 128 bytes).

hop network topology is better from a region where relaying schemes (cooperation or multi-hop) are useful for energy efficiency. We clearly observe that the single-hop scenario is the most energy efficient network $(80 \%)$ when the transmit power exceeds $-4 \mathrm{dBm}$. Further, the single-hop scheme is twice as efficient as the two-hop scheme (40\%). Keeping in mind that the sensor nodes in the cow's WBAN are designed to work with low power values to extend the battery lifetime, the cooperative and two-hop scenarios present an energy efficiency larger than the single-hop scheme. For example, a transmit power of $-5 \mathrm{dBm}$ ensures an energy efficiency of $40 \%$ and $35 \%$ for two-hop and cooperative communications, respectively. However, energy efficiency is even less than $10 \%$ in the single-hop case.

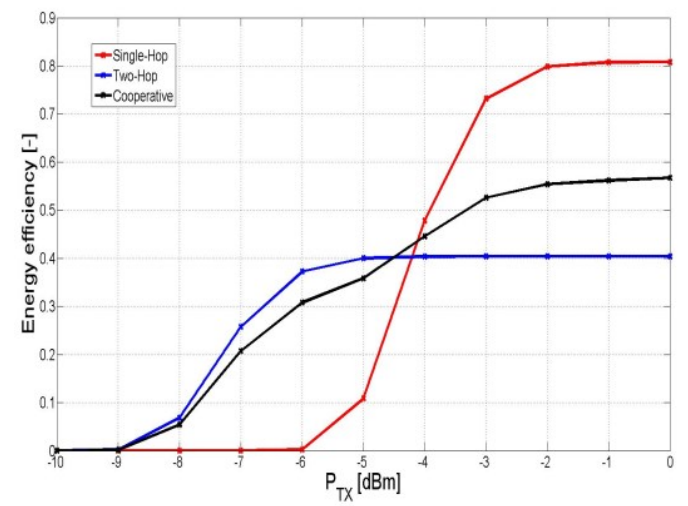

Fig. 8. Energy efficiency as a function of the transmit power $[\mathrm{dBm}]$ for the investigated communication schemes (packet size 128 bytes).

\section{CONCLUSIONS}

In this work, propagation modelling for dairy cows in barn environment has been presented. Both on-body and offbody wireless communications have been addressed. Path loss was found to be described by a one-slope path-loss model. Temporal fading was found to correspond excellently to Rician distribution with a $\mathrm{K}$-factor of $10 \mathrm{~dB}$. The physical propagation analysis have been used to design a cow-WBAN based on single-hop, multi-hop, and cooperative schemes.
The packet error rate and the energy efficiency a function of the sensor node transmit power have shown that multi-hop and cooperative communications allow a reduction of the power consumption, which would extend the lifetime of the dairy cow monitoring system.

\section{ACKNOWLEDGMENT}

This work was supported by the iMinds-MoniCow project, co-funded by iMinds, a research institute founded by the Flemish Government in 2004, and the involved companies and institutions. E. Tanghe is a Postdoctoral Fellow of the FWO-V (Research Foundation - Flanders). The authors would like to thank Thijs Decroos, Matthias Van den Bossche, and Sara Van Lembergen for helping us during the measurements.

\section{REFERENCES}

[1] S. Benaissa, D. Plets, E. Tanghe, G. Vermeeren, L. Martens, B. Sonck, T. Frank, L. Vandaele, J. Hoebeke, and W. Joseph, "Characterization of the On-Body Path Loss at $2.45 \mathrm{GHz}$ and Energy Efficient WBAN design for Dairy Cows," IEEE Trans. Antennas Propag., vol. 127, no. 99, pp. 593-605, 2016.

[2] A. Schlageter-Tello, E. A. M. Bokkers, P. W. G. G. Koerkamp, T. Van Hertem, S. Viazzi, C. E. B. Romanini, I. Halachmi, C. Bahr, D. Berckmans, and K. Lokhorst, "Manual and automatic locomotion scoring systems in dairy cows: A review," Preventive Veterinary Medicine, vol. 116, no. 1-2. pp. 12-25, 2014.

[3] S. Gabriel, R. W. Lau, and C. Gabriel, "The dielectric properties of biological tissues: III. Parametric models for the dielectric spectrum of tissues.," Phys. Med. Biol., vol. 41, no. 11, pp. 2271-2293, 1996.

[4] J. B. Schneider, "Understanding the Finite-Difference Time-Domain Method," Self Publ., 2013.

[5] A. Andersen, "Texas Instruments: Design Note $007-2.4 \mathrm{GHz}$ Inverted F Antenna," 2008.

[6] E. Reusens, W. Joseph, B. Latre, B. Braem, G. Vermeeren, E. Tanghe, L. Martens, I. Moerman, and C. Blondia, "Characterization of On-Body Communication Channel and Energy Efficient Topology Design for Wireless Body Area Networks," Inf. Technol. Biomed. IEEE Trans., vol. 13, no. 6, pp. 933-945, 2009.

[7] M. M. Khan, Q. H. Abbasi, A. Alomainy, and C. Parini, "Experimental Investigation of Subject-Specific On-Body Radio Propagation Channels for Body-Centric Wireless Communications," Electronics, vol. 3, no. 1, pp. 26-42, 2014.

[8] A. Abdi, C. Tepedelenlioglu, M. Kaveh, and G. Giannakis, "On the estimation of the $\mathrm{K}$ parameter for the rice fading distribution," IEEE Commun. Lett., vol. 5, no. 3, pp. 92-94, 2001.

[9] E. Tanghe, W. Joseph, L. Verloock, L. Martens, H. Capoen, K. Van Herwegen, and W. Vantomme, "The industrial indoor channel: Largescale and temporal fading at 900, 2400, and $5200 \mathrm{MHz}$," IEEE Trans. Wirel. Commun., vol. 7, no. 7, pp. 2740-2751, 2008.

[10] A. F. Molisch, Wireless communications, Second edi. wiley, 2011. 